\title{
Analytical Execution of Dynamic Routing Protocols For Video Conferencing Applications Based on OPNET Modeler
}

\author{
Dr Adnan Hussein Ali Haeeder Munther Noman Dr Hassan S. Hamad \\ Middle Technical University, Baghdad, Iraq/ Technical Instructors Training Institute
}

\begin{abstract}
-
In modern network communications, Routing protocols are getting an important function for the user data path that are responsible for controlling the routers to communicate together and forward packets by routers over the best trip path from a base node to a destination one. Dynamic routing protocols represented by RIP, OSPF and EIGRP are explained here for addressing various networks with different traffic environments. In this paper, the performance of these protocols are estimating with many factors like convergence activity and duration, average throughput, network end-to-end delay, Point-to-Point Utilization over the simulation based on OPNET academic version. From Simulation results, EIGRP have a fastest time convergence compared with other topologies of networks are confirmed and the OSPF has the highest Point-to-Point Utilization in the network followed by EIGRP then RIP. So, there is an attempt for finding out which protocols are suitable for the networks and from analyses to understand the role of the routing protocols in different network scenarios.
\end{abstract}

Keywords: RIP, OSPF, EIGRP, OPNET, convergence, and network performances.

Date of Submission: 27 February 2017

$\longrightarrow$

Date of Accepted: 11 March 2017

\section{INTRODUCTION}

In computer networks, an ultimate essential aspect for communication be the routing and it can be prepared with routing protocols, it assigns how routers are connect to choice the paths for data or information transferring for that, and a routing algorithm considered very essential, Firstly, the routing protocol notifies or portions the data or information with other associating neighbors and also through the network, in which topology [1]. Routing is categorized into two major classifications with respect to form a routing board, Static and Dynamic routing. Static routing supposes a fixed network, which means no nodes must be added or extracted as well as routing tables be consequently can updated manually [2].

Dynamic routing shows a significant part in the current networks, they are utilized to simplify interchanging the information of routing among routers, also agrees changes in the topology of networks by utilizing routing tables which can be updating by changing each network, and controlling the greatest path to the destination. By comparing with static routing, the dynamic routing protocols improve the scalable and adaptable behaviors as well as involve less executive overhead. Dynamic routing techniques will be capable for sustaining the procedure of networks in failure situation or any change in network topology [3].

In the context group of dynamic protocols, there is an Interior Gateway (IGP) or Exterior Gateway Protocols (EGP) can be found, EGP's concentrated on routing information during various Autonomous, like Border Gateway Protocols (BGP). The interior gateway (IGP) routing protocols represented by Distance vector like RIP which means Routing Information Protocol and EIGRP that means Enhanced Interior Gateway Routing Protocol, and link state algorithms like OSPF, Open Shortest Path First of the Routing Protocol and hybrid protocol [4].

The IGP forms (Distance vector and link state) can be used for characterizing routing protocols utilized to forward packets by routers. These two types of routing protocols, shown in fig. 1, centered on two cases, first if routing protocol chooses the better path of routing created on (the distance), while the other focused on an interface or (the vector) which chooses the better path of routing by finding each link state within a path and then to find any path with least total metric at the Destination [5]. 


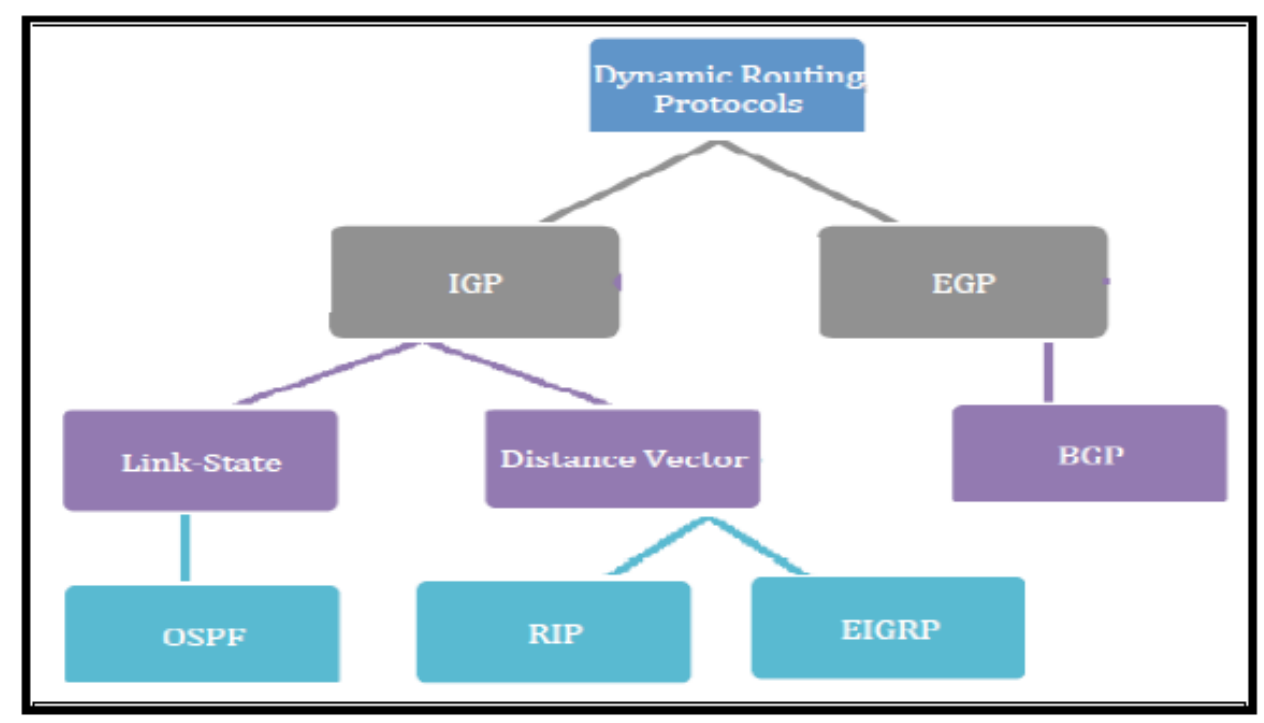

Fig.1: types of dynamic routing protocols

Protocol like Distance vector estimates the best path established on distance, it measures with expressions of hops or set of metrics evaluated for representing the distance value. The most Internet Protocol IP Distance protocols for the time being are RIP and IGRP. While for state routing, all nodes construct a map for connection to a network with a form of a graph viewing a connection of nodes with each, then each node autonomously determines the following path (the best logical one) to every potential destination inside the network [6]. The table of node's routing can be formed with a collection of best paths.

\subsection{Routing Information Protocol (RIP)}

\section{OVERVIEW OF DYNAMIC ROUTING PROTOCOLS}

RIP is consider protocol for LAN and WAN and related to interior gateway routing by which distance vector can be utilized for data/packet transmissions and Hop count as its metric, also its originally first protocol that can executed on the TCP/IP [7]. Typical RIP extremely shows 16 hops count from sources to destination thus offer loop free routing and size limit network. In RIP organized networks, Request Message in addition to the Response Message can be send as well as received by routers from another RIP organized routers within the network by a constant period of time.

The largely acceptance of RIP protocols are owing to their simplicity and easy configuration. RIP have four components namely, RIP metric, RIP timers, RIP stability and RIP updating processes. RIP protocol utilizes group of timers can be considered as very important component called an update timers, true timers and hold down timers used for convergence activity, but if topology be changed then manual updating not necessary. RIPs initial configuration of the network have advantages like simplicity, and consider as a routing protocol which have poor convergence activity and restricted hop counts, on the other hand, RIP deals with less CPU utilization [8].

\section{2 (OSPF) Routing Protocol}

OSPF considers a well-known normally applied link states internal gateway protocols (IGP). It means Internet Protocol packet with assembling information of link states getting with adjacent routers so that creating the network mapping. It introduced in mid-80`s by Internet Engineering for IP network. OSPF Protocols broadly utilize in large initiative networks due to their effective convergence [9]. In OSPF protocols, information of network topology distributes by routers inside the network. OSPF is consider dynamic routing protocol, therefore all routers which configured by OSPF protocol can interchange the information of link state routing with all connecting routers so that to build their specific routing table. Dsijktra algorithm is utilized for finding the shortest route inside the table route from an existing router to all connecting routers [10].

If there is a link failure or a setting in the network, then network routers come to be active so they will be convergence activity and each routers interchange topological link failure information toward the network. The routers of OSPF can direct abundant message forms comprising greetings messages, demands and updating link states, with database description. OSPF advantage is more flexible and strong protocol due to its structure of many configurable parameters. Comparing with RIP, OSPF protocol disadvantage is being so complicated.

In 1989, OSPF had the first version that defined as OSPFver1, The OSPFver2 was announced in 1998, create in RIFC 2328. In 1999, OSPFver3 was released for IPver6, available in RIFC 2740. 


\section{3 (EIGRP) Routing Protocol}

EIGR Protocol belong to routing of distance vectors which utilizes an algorithm named Diffusion Update Algorithm (DUAL). However, EIGRP is hybrid routing protocol owing to get also a properties of link state protocols [11]. That Means, EIGRP comprises the structures of link state in addition to distance vector protocols. EIGRP protocol typically deals with large networks. Each EIGRP routers within the network keep all of them routes in their routing table. It also makes convergence when topological changes occur in the network. If a router is incapable for finding a path over the network, then a query will be send out to all neighbors, so it spreads until an appropriate route be found.

EIGRP protocol procedure is established with four modules that are Neighbor Discovery Recovery, DUAL, Reliable Transport Protocol (RTP), and dependent module Protocol [12]. The first one, Neighbor discovery protocol is one of the routes of EIGRP protocol by which the router determines the neighboring routers with sending packets HELLO with fixed period of time. RTP the second confirms the consistent transmission of EIGRP's unicast or multicast packets of protocol in the network. DUAL shows a significant part as a loop avoidance mechanism that may be utilized for the topological change within the network.

When a failure or set of any link within the network which means there is a topological change is happened then DUAL protocol will be activated with EIGRP protocol by means of convergence activity. As a consequence of DUAL, the routers will be updating their routing table. EIGRP protocol exhibits loop free paths so that it becomes easy for configuration in huge networks, and it also can preserve the backup loop free route through the destination by the support of DUAL mechanism. EIGRP Protocol that following Modules assists to figure the topology table that is centered on DUAL mechanism. EIGRP protocol backs Cisco router alone which it means only suitable with a Cisco technology so that other routers will not exploit EIGRP protocol.

\section{RELATED WORK}

Ahmed et al, 2008 [13], had been used different algorithms to illustrate OSPFWS problems and discussed each algorithms and explained the advantages and disadvantages of algorithms. As result was each algorithms may be better by comparing with another according to the application of papers, and finally they created new suggestion for determining the OSPWS problems. A. Gani et al. 2011[14], they suggested a new routine to improve the RIP efficiency with including an intelligence component. Their planned technique assists preliminary step for creating a network with self-configured. Karamjeet et al. 2012 [15], found the best route by using simulation for OSPF. Their work applied on wired and wireless LANs with performing several sorts of simulations and focused on OPNET. The networks were designed in OPNET to achieve OSPF protocol operation and result of simulation assigned OPNET modeler to assess the execution for the wireless network protocol containing OSPF. Pankaj et al. 2012[16], here analyzing the execution for the different routing like OSPF, RIP, IGRP \& EIGRP protocols were shown based on transmission cost, delay and router throughput. OSPf can transmit a packet better than others. G.C Nwalozie et al. 2012 [17], explained the OSPF performance and implementation in addition to the predictable route RIP with an improved type. They show OSPF that used for optimizing the performance metrics for networks with using dijkestra procedure to evaluate the lack path toward the target networks.

V. Vetriselvan et al 2014. [18], here, routing protocols had typical challenges and discussed approximation of numerous routing protocol issues that were assorted for many routing protocols if they were utilized with actual conditions. Md Samil et al. 2014[19], had made a comparison between RIP and OSPF protocols with a theoretical fact. OSPF accomplishes a throughput and instant packet delay of RIP and its coverage network more quickly than RIP, so, RIP is good with FS-RIP, and shows a best performance with respect than OSPF. A. Verma and N. Bhardwaj (2016)[20] give a comparative study for the most elective RIP and OSPF protocols and getting best route in wired and wireless LANs for them.

\section{NETWORK STRUCTURES AND EXECUTION TOOL}

For designing networks, a discussion of numerous mechanisms for utilizing networks in addition to all functions. All suggested components will be seen in the building of networks and employment with OPNET, they are:

Applications Configr: the node that is utilized for setting the applications in any networks and can be utilized for Ace group Information, browsing of web represented by full http, and scheme encoder of voice specifications.

Profile Configr: the nodes that give describe of applications and can achieve managements. They are a user profile which made at any node can be utilized in various nodes at network for generating traffic in application layer. These Profile Configr can be utilized to describe a traffic manners which is pursued with these applications.

Particular configurations for IP that established a model of router gateway. Their specifications are: 
Ethernet_wrkstn: node model that symbolizes client-server applications in a workstation operating over UDP/IPr and TCP/IPr. The wrkstn are backing any basic connection with $10 \mathrm{mmbps}$, 100mmbs and 1000mmbps.

PPP_DSS3: it's a full duplex link that link twice IPs nodes.

100sBaseT: its full duplex connections that can be used for signifying the connections of Ethernet. It used for connecting any arrangement of nodes like Stations, Bridges, Switches, Hub and LLAN nodes.

Failures Recovery: it's a controlling nodes which is utilized for failure-recover models.

\section{Scenarios}

The system is simulated by OPNET modeler. The application is prepared for three routing protocols which are RIP, OSPF and EIGRP. A simulation is done with three main scenarios.

Scenario A be designed as a RIP protocol Baselines scenarios. While Scenario B is an OSPF protocol Baselines. Finally Scenario C is designed for EIGRP protocols. In the network model, five Cisco 7000 routers will be using in addition to two PCS as a Work stations, Profile Config, Application Config, with a Link Failure. For comparing the results with our three scenarios, a model of baseline network containing of five routers Cisco 7000 are connected together through ppp_dss3 connections with twice Ethernets_wrkstn and twice PCS that could represented videos conferencing wrkstns as shown in fig. 2.

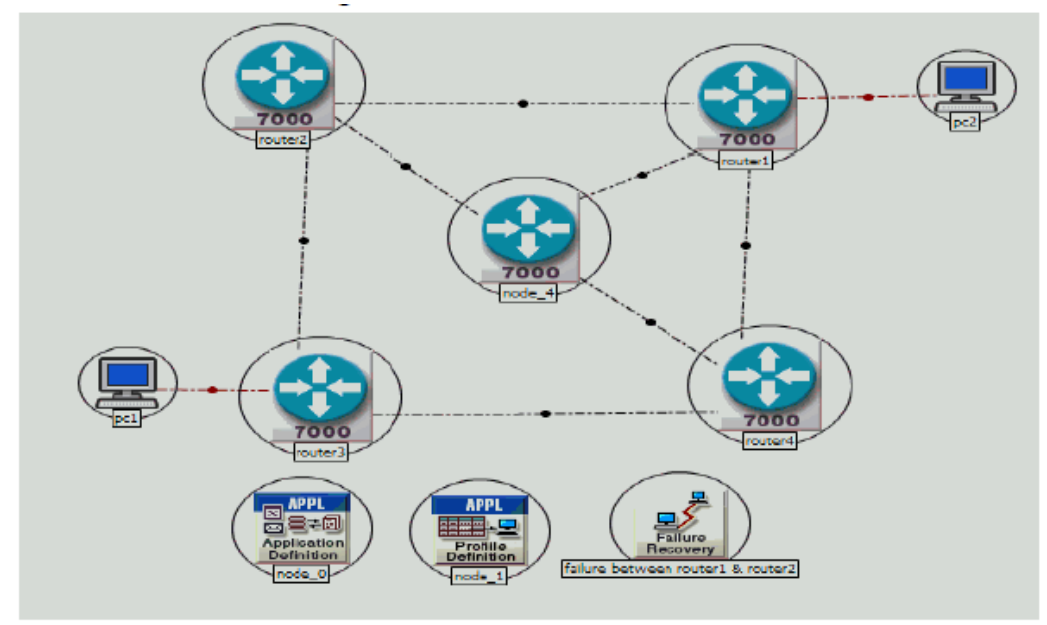

Fig-2: the suggested network

\section{Comparison between routing protocols}

By using OPNET modeler as a networking tool used for simulations, three network scenarios are being designed having the same specification but only the routing protocol will be changed during the simulation. OPNET presents a networking medium that achieves the requirements to simulate the model of communication networks. In order to show a comparison in each network, the link between R1 and R2 will be tested with two tables of different states of fail and recovery as shown in tables $1 \& 2$.

\begin{tabular}{|c|c|}
\hline fail & recover \\
\hline 240 & 480 \\
\hline 720 & 960 \\
\hline 1200 & 1440 \\
\hline \multicolumn{2}{|c|}{ Table-1: state A fail \& recovery } \\
\hline fail & recover \\
\hline 240 & 420 \\
\hline 520 & 580 \\
\hline 610 & 620 \\
\hline 625 & 626 \\
\hline 726 & 826 \\
\hline
\end{tabular}

Table-2: state B fail \& recovery 


\section{SIMULATION RESULTS \& DISCUSSION}

The routing protocols that are proposed with many scenarios in the network (RIP, OFPF, and EIGRP) are be compared for the two suggested states, based on Convergence Activity, convergence length, packet ends to ends delay, the average point-to-point throughput, and an average point-to-point utilization while running video conferencing as a real time applications.

\section{Convergence Activity}

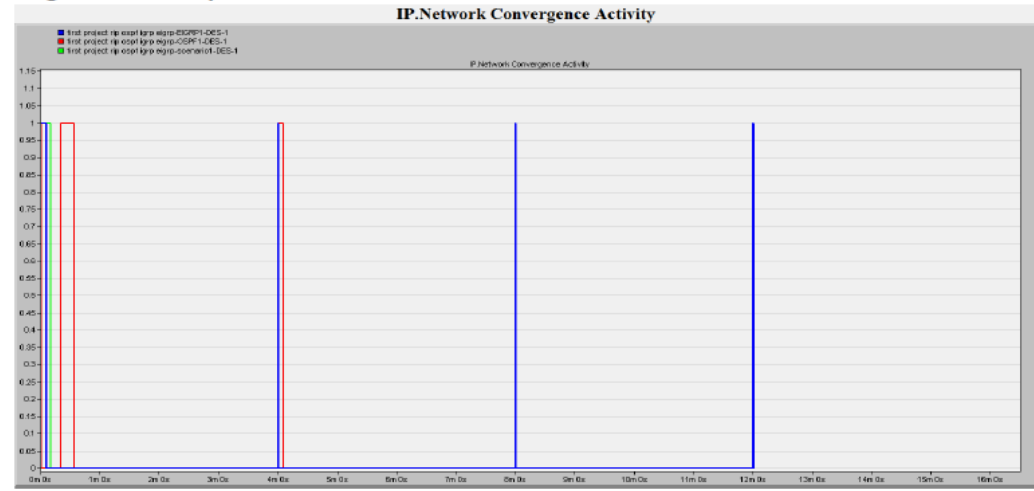

Fig. 3 Convergence Activity for state A

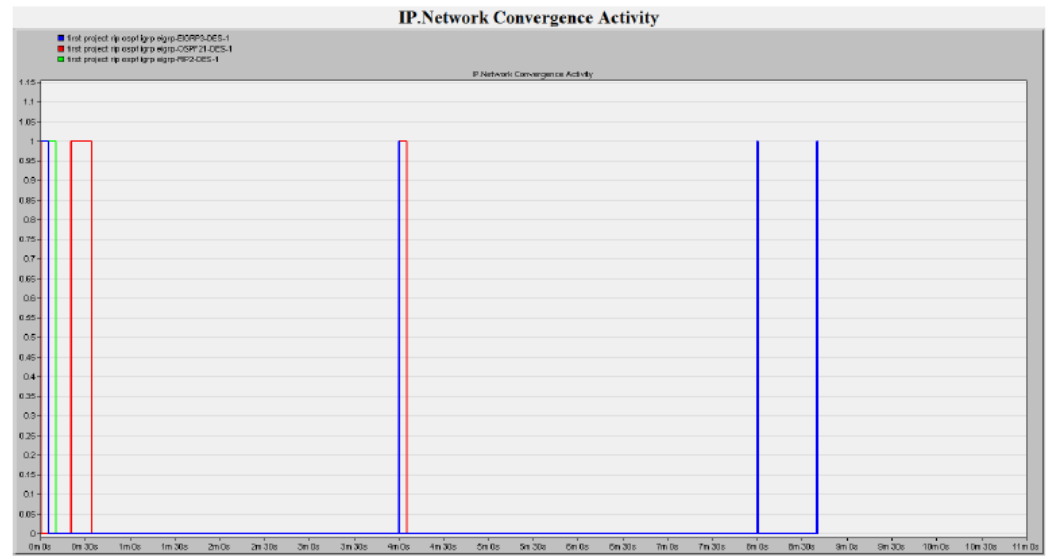

Fig .4 Convergence Activity for state B

Fig. 3, 4 show the average network convergence activity for RIP, OSPF and EIGRP for the two states respectively. The first one (red), seconds (blue), with a thirds one is (green) peaks explain the starting situation, the links failures will be 240 secs, with a links recovery can be seen at 480 s secs. Any peaks have a width which illustrates the convergence duration. If a routing protocol is longer period for convergence, then a high width peak should be. By observing these results, we see that OSPF have the slowest activity convergences, a red peak have bigger width that show the OSPF is slow compare to RIP and EIGRP, and the blue peak represent EIGRP which has a smaller width that means it has the fastest convergence activity.

\section{Convergence Duration}

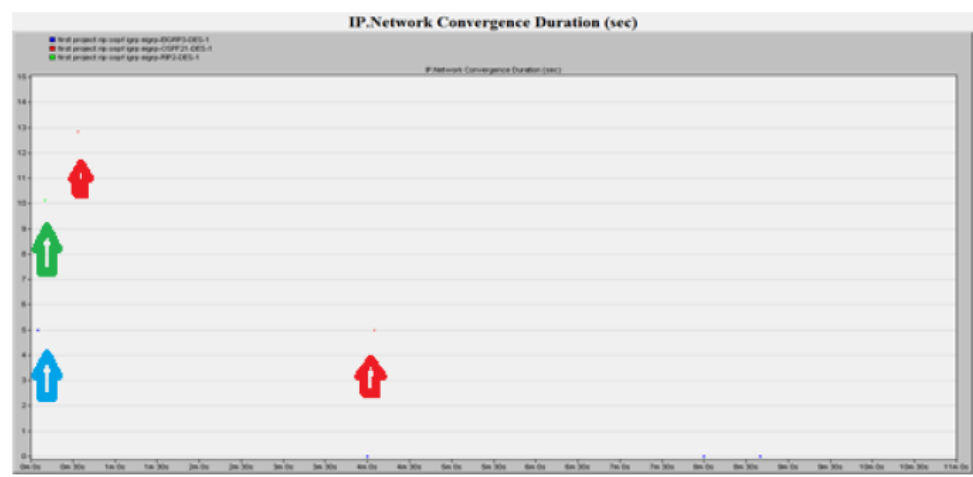

Fig-5- convergence duration for state A 


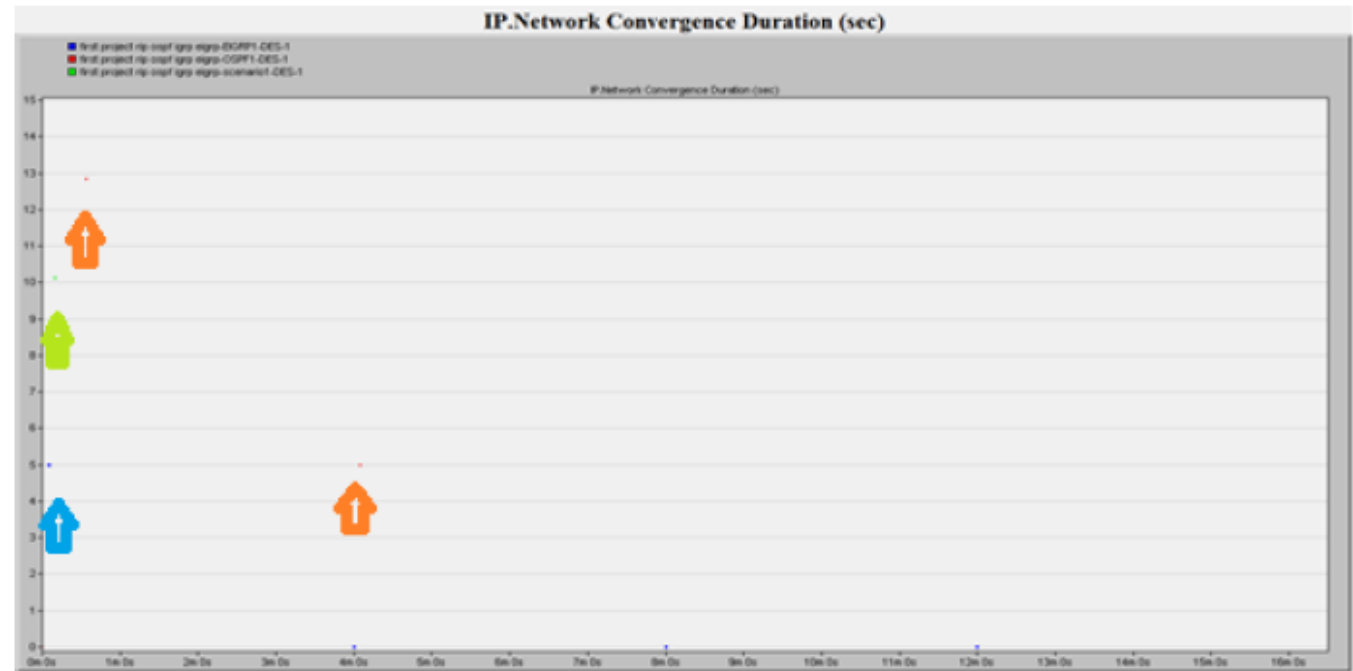

Fig-6- convergence duration for state B

Figures 5, 6 show the average network convergence duration between RIP, OSPF and EIGRP for the two states respectively. The fastest network convergence duration goes to the EIGRP (Blue line) which approach to zero seconds. RIP (green line ) in our scenario converges faster than OSPF (red line) because the link failure is just one hop far so RIP has less convergence duration time then OSPF, in addition to that OSPF is link state protocol which depends on topology database flooded throughout the network area.

\section{Packet End to End Delay}

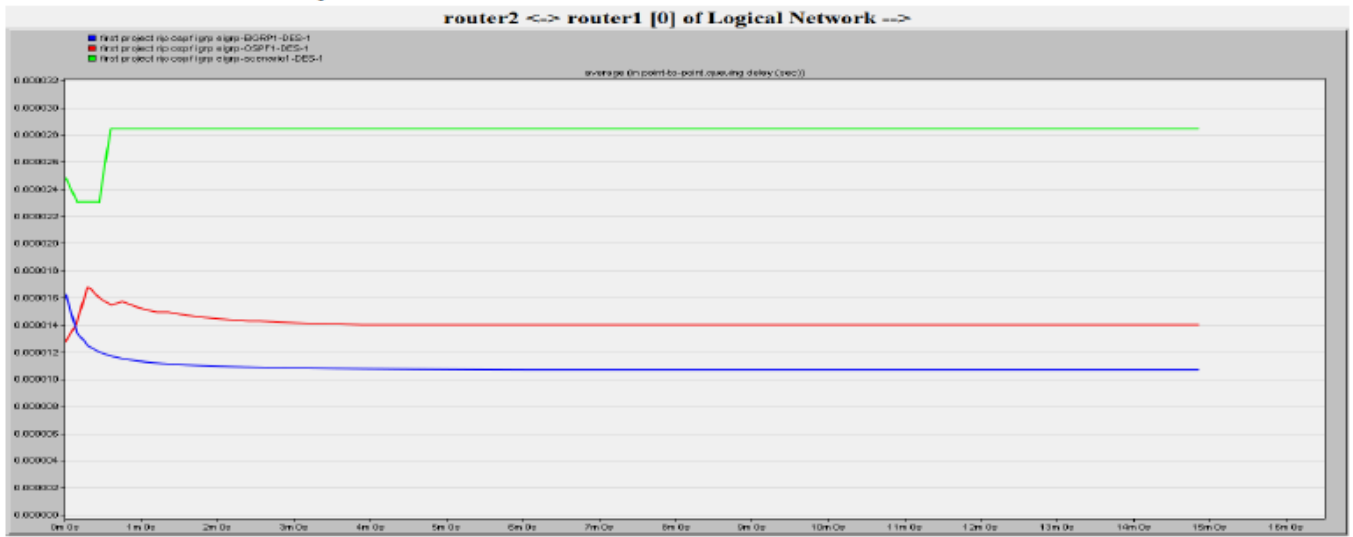

Fig-7- packet end to end delay for state A

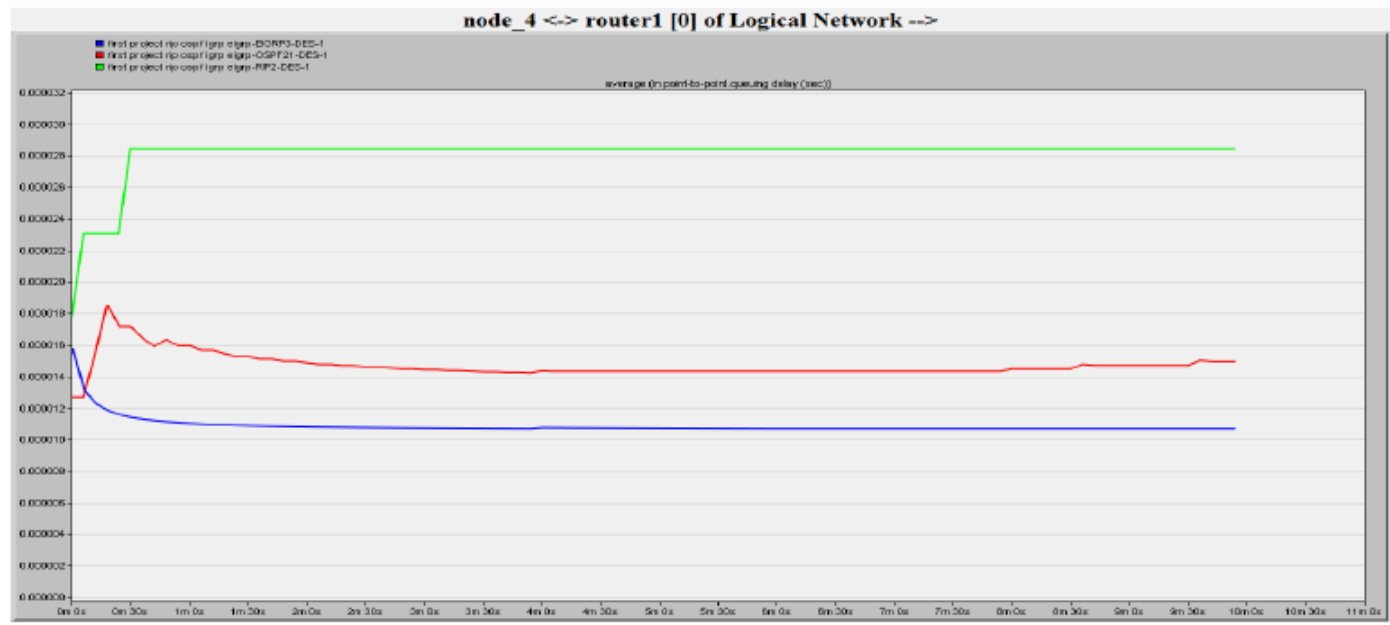

Fig-8- packet end -to- end delay for state B 
The ends-to- ends delay of all three scenarios shown in figures 7and 8, the higher delay can be seen for RIP (represented by green line), it's clearly show the lack of intelligence in the RIP protocol, the OSPF (represented by red line) start less delay than the EIGRP (blue line) but then increases while EIGRP starts higher and then decreases with time goes on. So in the network where EIGRP is implemented, less delay is obtained.

\section{Points-to-Points Throughputs}

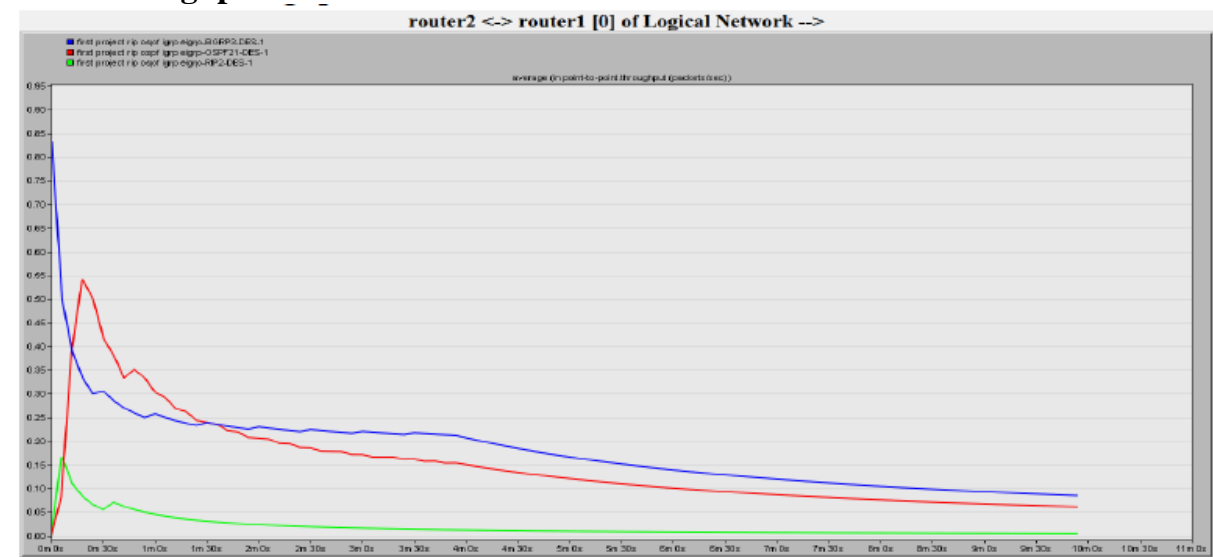

Fig-9- average point -to- point throughput for state A

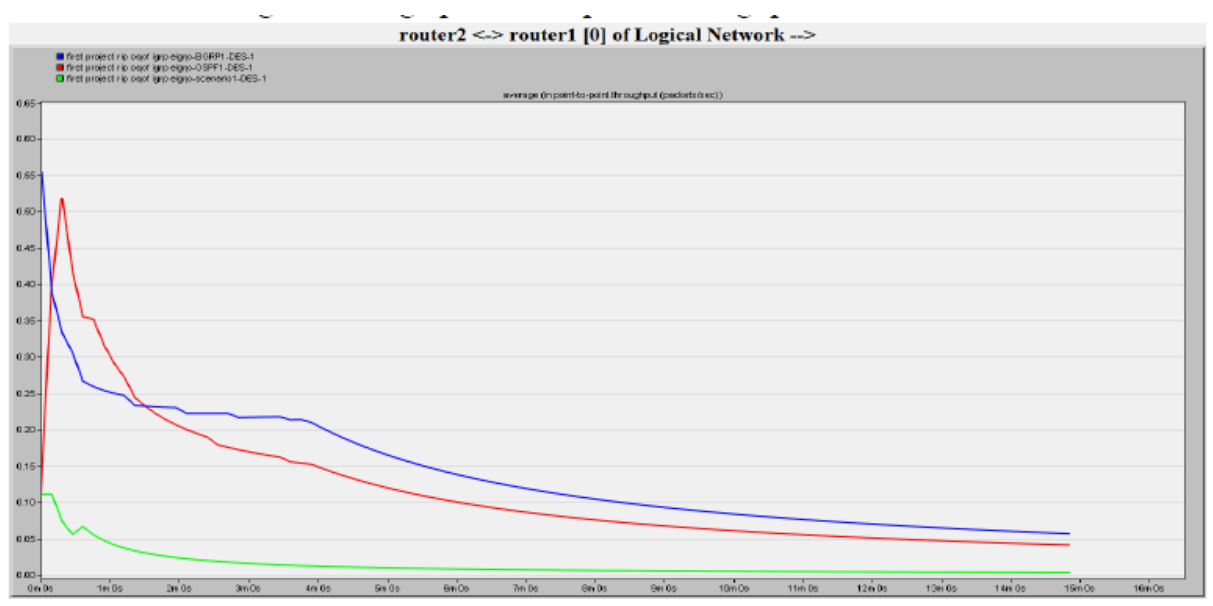

Fig-10- average point -to- point throughput for state B

The averages points-to- point throughputs can be shown in figure 9, the EIGRP (blue line) has high throughput in the first minute and stabilize with time goes on, just because the protocol learn the network topology in the beginning it send high date rate. The RIP (green line) has the less point-to-point throughput.

\section{Points-to-Points Utilizations}

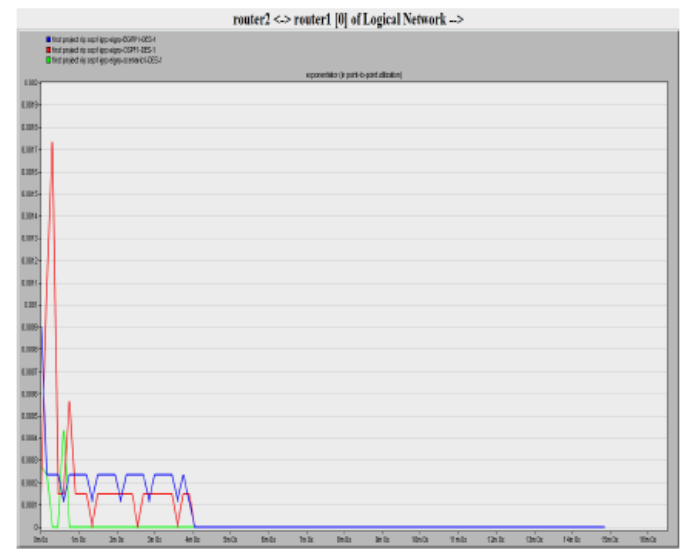

Fig-11- Point -to-Point Utilization for state A

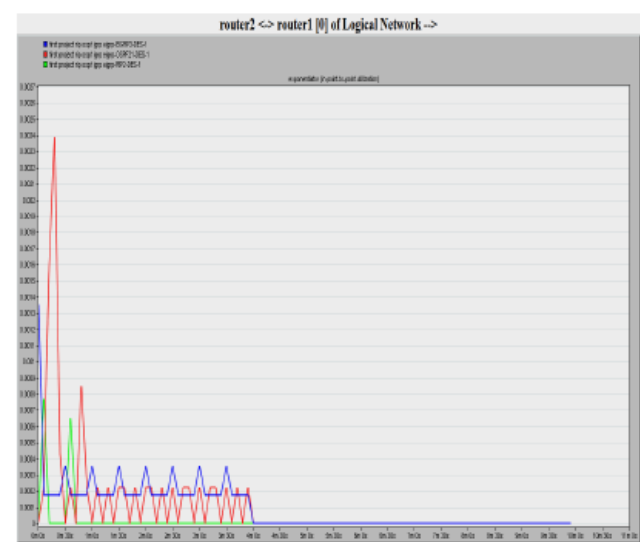

Fig-12- Point -to-Point Utilization for state B 
The average point-to-point utilization can be shown in fig $11 \& 12$, the OSPF has the highest Point-to-Point Utilization in the network followed by EIGRP then RIP.

\section{CONCLUSION}

For comparing the execution to three most popular dynamic routing protocols, a results of network convergence, throughput, end-to-end delay, and utilization are analyzed theoretically and graph simulations. Outcomes of Simulation are proven that, the EIGRPs have a fast period of convergences to the all kinds of networks. It has also been noted here, EIGRPs and OSPFs are all proficiently operate the bandwidth because both protocols do not periodically send updates as the case of RIP protocol. The RIPs transmits all routings information over periodic updates, which overflow the network in addition to needlessly losses of bandwidth, finally the OSPF has the highest Point-to-Point Utilization in the network followed by EIGRP and RIP.

\section{REFERENCES}

[1]. Behrouza A. Forouzann, “TCPP/IPP Protocol Suiter”, McGrawr- Hilll Education Presss. P. 269. ISBN 0-037-37064-3. Retreived on March 25, 2009.

[2]. D. Anveshini, S. Pallam Shetty, "Analyzing the steady state behaviors of RIPs and OSPFs routing protocols ina the contexts of linkd failures and link recovery ina Wide Area network", International Journal of Computer \& Organization Trends (IJCOT)Volume 34 Number2- August 2016, ISSN: 2249-2593.

[3]. Sheh, A. and Waqqas, Jour. "Performances Analyses of RIPs and OSPFs in Networks Using OPNETT" International Jour. off Computer Sciences (2013).

[4]. Yang1, S. and Zhis Yang, Z. “RIPs Internets Protocols Failurer Analyses and Researches” 2012 International Conferences on Industrials Controll and Electricals Engineerings (2016).

[5]. Rakkeja, P., kuar, P., guptas, A. \& Sharmas, A. "Performances Analyses of RIPs, OSPFs, IGRPs and EIGRPs Routingg Protocols in an Networks". Int. Jour. off Computers Applications, vol.48, no.(18), pp. 16-11, (2012).

[6]. Fiţigăur, I. and Todereano, G."Networks Performances Evaluations for RIPs, OSPFs and EIGRPs RRouting Protocols (2016).

[7]. Simon Fraser University, finall projects, "Analysis off RIPs, OSPFs, and EIGRPs Routingg Protocols usings OPNETT", springer 2013.

[8]. Expósitos, J., Trujillos, V. and Games, E. "A Didactics Applications ford Teachings and Learnings off then Enhanced Interiors Gateways Routing Protocols", 2010 Sixth Int. Conf. on Networks and Service. An Easy -EIGRPs", 2010.

[9]. M. Jafar, T., F. Rileys, G., Roddy, D. and Bliar, D. "Simulation- Based Routings Protocoling Performances Analyses“(2006). Proceedings off the 2006 Winters Simulations Conferences.

[10]. J. Kumaro, M.," An comparatives studying on RIPs and OSPFs protocols", Inventions in statistics, an Embedded and conversations structures (ICIIECS), pp. 1-5, 2015.

[11]. M. Masoodd, M. Abduhelala, "An comprehensives study off Routings Protocols Performances with Topologically Changes in then Networks", Int. Jour. off Electronics, Electrical and Computational Systems (IJECES), Vol. 5, Issue 8, August 2016.

[12]. Noman, A. and Chowdhury, A. "Performances Analysis off EIGRPs and OSPFs ford Different Applications using OPNETT". Australians J. of Comp. Sciences, vol.1, no. (1), pp.01-08, 2014.

[13]. As. G. Ahmed, E. Aymani \& M. Mervats, "An Surveys ford Open Shortest Path First Weight Setting (OSPFWS) Problems", Int. Conf. on Information Security \& Assurance, (2008).

[14]. A. Ganid, M. Hassan, A. Zaidany and B. Zaidany, "Intelligent routings information protocols using full Trigered up-date mechanisms", Int. Jour. off then Physical Sciences, v. 6, no. 11, (2011).

[15]. K. Kauri, S. Singha and R. Malhorta, "Designs off Open Shortest Path First Protocols - An Link States Protocol Using Opnett Modulator", IJCSMMC, volume 1, no. 01, (2012).

[16]. P. Rakeja, P. Kauri, A. Gurpta and A. Sharmah, "Performances Analysis off RIPs, OSPFs, IGRPs and EIGRPs Routing Protocols in an Networks", Int. Jour. of Computers Applications, volume 48, no.18, (2012).

[17]. G. C. Nwalozic, V. N. Okoragu, A.C. Okaford and A.O. Umehi, "Evaluations And Implementations off then Open Shortest Paths First (OSPFs) Routings Protocols", Certified Journal, vol. 22, no. 112, (2012).

[18]. V. Vetrislvan, P. R. Patils and M. Mahendrani, "Surveys on then RIPs, OSPFs, EIGRPs Routings Protocols", Int. Jour. of Computers Sciences and Informational Technologies, volume 5, no. 2, (2014).

[19]. M. S. Farouqi, V. Nigama and G. Gandhii, "Evolutions off then Routings Protocol from RIPs (11969) ton OSPFs (1998)", IJARCCSE, volume 4, no. 2, 2014.

[20]. A. Vermah and N. Bardwaj, "An Reviews on Routings Information Protocols (RIPs) and Open Shortests Path First (OSPFs) Routings Protocols", Int. Jour. of Futures Generations Communications and Net-working Volume 9, No. 42016.

\section{AUTHOR'S PROFILE}

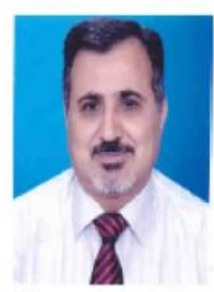

The Author is a Lecturer in the Computer Engineering Department at Institute of Technology. Baghdad, IRAQ. He has been awarded a Doctor of Philosophy in Laser and Opto-Electronics Engineering from University of Technology, Baghdad, in 2007. He has studied Master of Science in Electronics Engineering, Cupper Vapor Laser's Power supply at University of Technology, Baghdad in 2000. He has gained Bachelor in Electrical and Electronic Engineering from University of Technology, Baghdad, in 1987. Currently he is lecturer at Institute of Technology, Baghdad, IRAQ. His research interests are Radio over Fiber, Wireless Network, Laser's Power supply and OPNET.

\section{AUTHOR'S PROFILE}

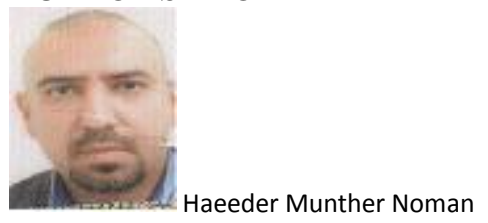

The author is an assistant lecturer in a department of electrical techniques in technical instructors training institute, Baghdad, Iraq. He holds a master degree in information technology engineering from GGSIP University in India in 2010. 